\title{
Hardy-Sobolev Spaces Associated with Twisted Convolution
}

\author{
Jizheng Huang, Weiwei Li, and Yaqiong Wang \\ College of Sciences, North China University of Technology, Beijing 100144, China \\ Correspondence should be addressed to Jizheng Huang; hjzheng@163.com
}

Received 15 March 2017; Revised 21 May 2017; Accepted 28 May 2017; Published 21 June 2017

Academic Editor: Alberto Fiorenza

Copyright (C) 2017 Jizheng Huang et al. This is an open access article distributed under the Creative Commons Attribution License, which permits unrestricted use, distribution, and reproduction in any medium, provided the original work is properly cited.

We first define the Hardy-Sobolev spaces associated with twisted convolution; then we give the atomic decomposition. As an application, we consider the endpoint version of the div-curl theorem for the twisted convolution.

\section{Introduction}

It is a well-established fact that, for the purposes of harmonic analysis or theory of partial differential equations, the right substitute for $L^{p}\left(\mathbb{R}^{d}\right)$ in case $p \in(0,1]$ is the (real) Hardy space $H^{p}\left(\mathbb{R}^{d}\right)$, or its local version $h^{p}\left(\mathbb{R}^{d}\right)$ (cf. [1]). The Hardy spaces, or their local versions if needed, behave nicely under the action of regular singular integrals or pseudodifferential operators. Moreover, in the case of Hardy spaces the Littlewood-Paley theory and interpolation results extend to the whole scale of Lebesgue exponents $p \in(0, \infty)$. It is hence natural to investigate Sobolev spaces where one (roughly speaking) demands that the sth derivative belongs to a Hardy type space in the case $p \leq 1$. After the fundamental work of Fefferman and Stein [2] this line of research was initiated by Peetre in early 70s, and it was generalized and carried further by Triebel and others. We refer to $[3,4]$ for extensive accounts on general Besov and Triebel type scales of function spaces in the case $p \in(0,1]$.

Let $I_{\alpha}: \mathcal{S}^{\prime} / \mathscr{P} \rightarrow \mathcal{S}^{\prime} / \mathscr{P}$ be the Riesz potential operators, where $\mathcal{S}^{\prime}$ is the space of tempered distributions and $\mathscr{P}$ denotes the space of polynomials. We can define Sobolev space $I_{\alpha}\left(L^{p}\right)(p>1)$ to be the space of tempered distributions having derivatives of order $\alpha$ in $L^{p}$. The use of the HardySobolev spaces gives strong boundedness of some linear operators instead of the weak boundedness. For instance this is the case of the square root of the Laplace operator $\Delta^{1 / 2}$. The Hardy-Sobolev spaces were studied by many authors. In [5], the author investigated the spaces $I_{\alpha}\left(H^{p}\right)(0<p \leq 1)$, where $H^{p}$ denotes the Hardy spaces. The spaces $H^{p}$ form a natural continuation of the $L^{p}$ space to $0<p \leq 1$, and so the spaces $I_{\alpha}\left(H^{p}\right)$ which are called Hardy-Sobolev spaces are natural generalizations of the homogeneous Sobolev spaces $I_{\alpha}\left(L^{p}\right)$ to the range $0<p \leq 1$. Strichartz [5] proved that $I_{n / p}\left(H^{p}\right)$ was an algebra and found equivalent norms for the Hardy-Sobolev spaces or, more generally, for corresponding spaces with fractional smoothness and Lebesgue exponents in the range $p>n /(n+1)$. Torchinsky [6] discussed the trace properties of the spaces $I_{\alpha}\left(H^{p}\right)$. Miyachi [7] characterized the Hardy-Sobolev spaces in terms of maximal functions related to mean oscillation of the function in cubes, thus obtaining a counterpart of previous results of Calderon and of the general theory of DeVore and Sharpley [8]. More recently there has been considerable interest in Hardy-Sobolev spaces and their variants on $\mathbb{R}^{d}$, or on subdomains. Chang et al. [9] consider Hardy-Sobolev spaces in connection with estimates for elliptic operators, whereas Auscher et al. [10] study these spaces with applications to square roots of elliptic operators. Koskela and Saksman [11] show that there is a simple strictly pointwise characterization of the Hardy-Sobolev spaces in terms of first differences. In [12], the authors gave the atomic decomposition of the Hardy-Sobolev space and proved the endpoint case of the div-curl theorem of [13]. Also the papers of Cho and Kim [14], Janson [15], and Orobitg [16] are related to the theme of the present paper. Recently, functional spaces associated with operators are considered by more and more mathematicians. In [17], the authors studied the Sobolev spaces associated with the twisted Laplacian and the Global well posedness of nonlinear Schrödinger equation. In [18], the authors defined the Hardy spaces associated with twisted 
Laplacian by the heat maximal function. They also gave the atomic decomposition and Riesz transform characterizations for the Hardy spaces. In this paper, we first define HardySobolev spaces associated with twisted Laplacian based on $[17,18]$ and then give the atomic decomposition of them. Finally, we give an application of the Hardy-Sobolev spaces associated with twisted Laplacian.

The paper is organized as follows. In Section 2, we give some results that we will use in the sequel; In Section 3, we prove some properties of the Hardy-Sobolev space, including atomic decomposition. In Section 4, some applications will be given.

\section{Preliminaries}

In this paper we consider the $2 n$ linear differential operators

$$
\begin{aligned}
& Z_{j}=\frac{\partial}{\partial z_{j}}+\frac{1}{4} \overline{z_{j}} \\
& \overline{Z_{j}}=\frac{\partial}{\partial \overline{z_{j}}}-\frac{1}{4} z_{j},
\end{aligned}
$$

$$
\text { on } \mathbb{C}^{n}, j=1,2, \ldots, n \text {. }
$$

Together with the identity they generate a Lie algebra $h^{n}$ which is isomorphic to the $2 n+1$ dimensional Heisenberg algebra. The only nontrivial commutation relations are

$$
\left[Z_{j}, \overline{Z_{j}}\right]=-\frac{1}{2} I, \quad j=1,2, \ldots, n .
$$

The operator $L$ defined by

$$
L=-\frac{1}{2} \sum_{j=1}^{n}\left(Z_{j} \overline{Z_{j}}+\overline{Z_{j}} Z_{j}\right)
$$

is nonnegative, self-adjoint, and elliptic. Therefore it generates a diffusion semigroup $\left\{T_{t}^{L}\right\}_{t>0}=\left\{e^{-t L}\right\}_{t>0}$. The operators in (1) generate a family of "twisted translations" $\tau_{w}$ on $\mathbb{C}^{n}$ defined on measurable functions by

$$
\begin{aligned}
\left(\tau_{w} f\right)(z) & =\exp \left(\frac{1}{2} \sum_{j=1}^{n}\left(w_{j} z_{j}+\overline{w_{j}} \overline{z_{j}}\right)\right) f(z) \\
& =f(z+w) \exp \left(\frac{i}{2} \operatorname{Im}(z \cdot \bar{w})\right) .
\end{aligned}
$$

The "twisted convolution" of two functions $f$ and $g$ on $\mathbb{C}^{n}$ can now be defined as

$$
\begin{aligned}
(f \times g)(z) & =\int_{\mathbb{C}^{n}} f(w) \tau_{-w} g(z) d w \\
& =\int_{\mathbb{C}^{n}} f(z-w) g(w) \bar{\omega}(z, w) d w
\end{aligned}
$$

where $\omega(z, w)=\exp ((i / 2) \operatorname{Im}(z \cdot \bar{w}))$. More about twisted convolution can be found in $[3,19,20]$.
In [18], the authors defined the Hardy space $H_{L}^{1}\left(\mathbb{C}^{n}\right)$ associated with twisted convolution. They gave several characterizations of $H_{L}^{1}\left(\mathbb{C}^{n}\right)$ via maximal functions, the atomic decomposition, and the behavior of the Riesz transform.

We first give some basic notations about $H_{L}^{1}\left(\mathbb{C}^{n}\right)$. Let $\mathscr{B}$ denote the class of $C^{\infty}$-functions $\varphi$ on $\mathbb{C}^{n}$, supported on the ball $B(0,1)$ such that $\|\varphi\|_{\infty} \leq 1$ and $\|\nabla \varphi\|_{\infty} \leq 2$. For $t>0$, let $\varphi_{t}(z)=t^{-2 n} \varphi(z / t)$. Given $\sigma>0,0<\sigma \leq+\infty$, and a tempered distribution $f$, define the grand maximal function

$$
M_{\sigma} f(z)=\sup _{\varphi \in \mathscr{B}} \sup _{0<t<\sigma}\left|\varphi_{t} \times f(z)\right| .
$$

Then the Hardy space $H_{L}^{1}\left(\mathbb{C}^{n}\right)$ can be defined by

$$
H_{L}^{1}\left(\mathbb{C}^{n}\right)=\left\{f \in \mathcal{S}^{\prime}\left(\mathbb{C}^{n}\right): M_{\infty} f \in L^{1}\left(\mathbb{C}^{n}\right)\right\}
$$

For any $f \in H_{L}^{1}\left(\mathbb{C}^{n}\right)$, define $\|f\|_{H_{L}^{1}\left(\mathbb{C}^{n}\right)}=\left\|M_{\infty} f\right\|_{L^{1}}:=$ $\left\|M_{\infty} f\right\|_{L^{1}\left(\mathbb{C}^{n}\right)}$.

Definition 1. Let $1 \leq q \leq \infty$. A function $a(z)$ is a $H_{L}^{1, q}$-atom for the Hardy space $H_{L}^{1}\left(\mathbb{C}^{n}\right)$ associated with a ball $B\left(z_{0}, r\right)$ if

(1) supp $a \subset B\left(z_{0}, r\right)$;

(2) $\|a\|_{q} \leq\left|B\left(z_{0}, r\right)\right|^{1 / q-1}$;

(3) $\int_{\mathbb{C}^{n}} a(w) \bar{\omega}\left(z_{0}, w\right) d w=0$.

We define the atomic Hardy space $H_{L}^{1, q}\left(\mathbb{C}^{n}\right)$ to be the set of all tempered distributions of the form $\sum_{j} \lambda_{j} a_{j}$, and the sum converges in the topology of $\mathcal{S}^{\prime}\left(\mathbb{C}^{n}\right)$, where $a_{j}$ are $H_{L}^{1, q}$-atoms and $\sum_{j}\left|\lambda_{j}\right|<+\infty$.

The atomic quasi-norm in $H_{L}^{1, q}\left(\mathbb{C}^{n}\right)$ is defined by

$$
\|f\|_{L \text {-atom }}=\inf \left\{\sum_{j}\left|\lambda_{j}\right|\right\},
$$

where the infimum is taken over all decompositions $f=$ $\sum_{j} \lambda_{j} a_{j}$ and $a_{j}$ are $H_{L}^{1, q}$-atoms.

Let $\psi$ be a $C^{\infty}$-function on $\mathbb{C}^{n}$ with compact support and such that $\psi \equiv 1$ on a neighborhood of zero. Define

$$
\begin{aligned}
& R_{j}(z)=\frac{z_{j}}{|z|^{2 n+1}} \psi(z), \\
& \bar{R}_{j}(z)=\frac{\bar{z}_{j}}{|z|^{2 n+1}} \psi(z)
\end{aligned}
$$

for $j=1,2, \ldots, n$.

We refer to the singular integral operators $R_{j}, \bar{R}_{j}$ defined by left twisted convolution with these kernels as the Riesz transforms. The terminology is motivated by the fact that they are essentially the operators which are formally defined as $Z_{j} L^{-1 / 2}, \bar{Z}_{j} L^{-1 / 2}, j=1,2, \ldots, n$.

The following result has been proved in [18]. 
Proposition 2. For a tempered distribution $f$ on $\mathbb{C}^{n}$, the following are equivalent:

(i) $M_{\infty} f \in L^{1}\left(\mathbb{C}^{n}\right)$;

(ii) for some $\sigma, 0<\sigma<+\infty, M_{\sigma} f \in L^{1}\left(\mathbb{C}^{n}\right)$;

(iii) for some radial function $\varphi \in \mathcal{S}$, such that $\int_{\mathbb{C}^{n}} \varphi(z) d z \neq$ 0 , we have

$$
\sup _{0<t<1}\left|\varphi_{t} \times f(z)\right| \in L^{1}\left(\mathbb{C}^{n}\right)
$$

(iv) $f$ can be decomposed as $f=\sum_{j} \lambda_{j} a_{j}$, where $a_{j}$ are $H_{L}^{1, q}$-atoms and $\sum_{j}\left|\lambda_{j}\right|<+\infty$.

(v) $f \in L^{1}\left(\mathbb{C}^{n}\right)$ and $R_{j} \times f, \bar{R}_{j} \times f \in L^{1}\left(\mathbb{C}^{n}\right)$ for $j=$ $1,2, \ldots, n$. [21].

Moreover, the following result has been proved in [18] or

Proposition 3. The Riesz transforms $R_{j}, \bar{R}_{j}, j=1,2, \ldots, n$, are bounded on $H_{L}^{1}\left(\mathbb{C}^{n}\right)$.

The dual space of Hardy space $H_{L}^{1}\left(\mathbb{C}^{n}\right)$ is defined in [18].

Definition 4. A locally integrable function $f$ is said to be in the $\mathrm{BMO}$ type space $\mathrm{BMO}_{L}$ if there exists a constant $K>0$ such that, for every ball $B=B(z, r)$,

$$
\begin{aligned}
& \frac{1}{|B|} \int_{B}\left|f(v)-\left(\frac{1}{|B|} \int_{B} f(u) \bar{\omega}(z, u) d u\right) \omega(z, v)\right| d v \\
& \quad \leq K .
\end{aligned}
$$

The norm $\|f\|_{\mathrm{BMO}_{L}}$ of $f$ is the least value of $K$ for the above inequality.

The Sobolev spaces associated with $L$ are defined as follows (cf. [17]).

Definition 5. Given $p \in(1, \infty)$ and $k \in \mathbb{N}$, we define the Sobolev space of order $k$ associated with twisted convolution, denoted by $W^{k, p}\left(\mathbb{C}^{n}\right)$, as the set of functions $f \in L^{p}\left(\mathbb{C}^{n}\right)$ such that

$$
\begin{aligned}
Z_{j_{1}} \cdots \bar{Z}_{j_{m}} f \in L^{p}\left(\mathbb{C}^{n}\right) & \\
& 1 \leq j_{1}, \ldots, j_{m} \leq n, 1 \leq m \leq k,
\end{aligned}
$$

with the norm

$$
\|f\|_{W^{k, p}}=\sum_{1 \leq j_{1}, \ldots, j_{m} \leq n, 1 \leq m \leq k}\left\|Z_{j_{1}} \cdots \bar{Z}_{j_{m}} f\right\|_{p}+\|f\|_{p} .
$$

Throughout the article, we will use $C$ to denote a positive constant, which is independent of main parameters and may be different at each occurrence. By $B_{1} \sim B_{2}$, we mean that there exists a constant $C>1$ such that $1 / C \leq B_{1} / B_{2} \leq C$.

\section{Hardy-Sobolev Spaces}

In this section, we define Hardy-Sobolev spaces associated with $L$ and consider some properties of them.
Definition 6. We define the Hardy-Sobolev space $H_{L}^{1,1}\left(\mathbb{C}^{n}\right)$ as the set of functions $f \in L^{1}\left(\mathbb{C}^{n}\right)$ such that

$$
\begin{aligned}
& Z_{j} f \in H_{L}^{1}\left(\mathbb{C}^{n}\right), \\
& \bar{Z}_{j} f \in H_{L}^{1}\left(\mathbb{C}^{n}\right), \\
& \quad j=1,2, \ldots, n,
\end{aligned}
$$

with the norm

$$
\|f\|_{H_{L}^{1,1}}=\sum_{j=1}^{n}\left(\left\|Z_{j} f\right\|_{H_{L}^{1}}+\left\|\bar{Z}_{j} f\right\|_{H_{L}^{1}}\right)+\|f\|_{L^{1}} .
$$

We can prove that $H_{L}^{1,1}\left(\mathbb{C}^{n}\right)$ is a Banach space. In order to do that, we need the following lemma (cf. P122 [22]).

Lemma 7. Let $1 \leq p<\infty, f \in L_{k}^{p}\left(\mathbb{C}^{n}\right)$, and $\left\{f_{m}\right\}$ be a sequence such that $\left\|f_{m}-f\right\|_{p} \rightarrow 0$. Then, for any $|\alpha| \leq k$, we have

$$
\left\|\frac{\partial^{\alpha} f_{m}}{\partial x^{\alpha}}-\frac{\partial^{\alpha} f}{\partial x^{\alpha}}\right\|_{p} \rightarrow 0
$$

By Lemma 7, we can prove the following.

Proposition 8. $H_{L}^{1,1}\left(\mathbb{C}^{n}\right)$ is a Banach space.

Proof. Let $\left\{f_{m}\right\}$ be a Cauchy sequence in $H_{L}^{1,1}\left(\mathbb{C}^{n}\right)$. Then $\left\{\partial f_{m} / \partial x_{j}\right\}$ and $\left\{x_{j} f_{m}\right\}, j=1,2, \ldots, n$ are Cauchy sequences in $H_{L}^{1}\left(\mathbb{C}^{n}\right)$. Let $f$ be the limit of $\left\{f_{m}\right\}$ in $L^{1}\left(\mathbb{C}^{n}\right)$. Then, by Lemma 7 ,

$$
\begin{gathered}
\left\|\frac{\partial f_{m}}{\partial x_{j}}-\frac{\partial f}{\partial x_{j}}\right\|_{1} \longrightarrow 0, \\
\left\|x_{j} f_{m}-x_{j} f\right\|_{1} \longrightarrow 0 .
\end{gathered}
$$

Since $H_{L}^{1}\left(\mathbb{C}^{n}\right)$ is a Banach space, there exist $g, h \in H_{L}^{1}\left(\mathbb{C}^{n}\right)$ such that

$$
\begin{gathered}
\left\|\frac{\partial f_{m}}{\partial x_{j}}-g\right\|_{H_{L}^{1}} \longrightarrow 0, \\
\left\|x_{j} f_{m}-h\right\|_{H_{L}^{1}} \longrightarrow 0 .
\end{gathered}
$$

By (17) and (18), we get $g=\partial f / \partial x_{j}$ and $h=x_{j} f$. This proves $\left\|Z_{j} f_{m}-Z_{j} f\right\|_{H_{L}^{1}} \rightarrow 0$ and $\left\|\bar{Z}_{j} f_{m}-\bar{Z}_{j} f\right\|_{H_{L}^{1}} \rightarrow 0$ for $j=$ $1,2, \ldots, n$, that is, $\left\|f_{m}-f\right\|_{H_{L}^{1,1}} \rightarrow 0$, and then we get $H_{L}^{1,1}\left(\mathbb{C}^{n}\right)$ is a Banach space.

Now, we give an equivalent characterization of $H_{L}^{1,1}\left(\mathbb{C}^{n}\right)$.

Definition 9. Let $\mathscr{H}_{L}^{1,1}\left(\mathbb{C}^{n}\right)=L^{-1 / 2}\left(H_{L}^{1}\left(\mathbb{C}^{n}\right)\right)$ or

$$
\mathscr{H}_{L}^{1,1}\left(\mathbb{C}^{n}\right)=\left\{f \in L^{1}\left(\mathbb{C}^{n}\right) \mid L^{1 / 2} f \in H_{L}^{1}\left(\mathbb{C}^{n}\right)\right\},
$$

with the norm $\|f\|_{\mathscr{H}_{L}^{1,1}}=\left\|L^{1 / 2} f\right\|_{H_{L}^{1}}+\|f\|_{1}$. 
Theorem 10. The norms $\|f\|_{H_{L}^{1,1}}$ and $\|f\|_{\mathscr{H}_{L}^{1,1}}$ are equivalent; that is, there exists a constant $C>0$ such that

$$
C^{-1}\|f\|_{H_{L}^{1,1}} \leq\|f\|_{\mathscr{H}_{L}^{1,1}} \leq C\|f\|_{H_{L}^{1,1}} .
$$

Proof. Let $f \in H_{L}^{1,1}\left(\mathbb{C}^{n}\right)$. Then, $Z_{j} f \in H_{L}^{1}\left(\mathbb{C}^{n}\right)$ and $\bar{Z}_{j} f \in$ $H_{L}^{1}\left(\mathbb{C}^{n}\right)$ for $j=1,2, \ldots, n$. Since $H_{L}^{1}\left(\mathbb{C}^{n}\right) \subset L^{1}\left(\mathbb{C}^{n}\right)$, we have $\left\|Z_{j} f\right\|_{1} \leq\left\|Z_{j} f\right\|_{H_{L}^{1}}$ and $\left\|\bar{Z}_{j} f\right\|_{1} \leq\left\|\bar{Z}_{j} f\right\|_{H_{L}^{1}}$ for $j=1,2, \ldots, n$. Note that $Z_{j}=Z_{j} L^{-1 / 2} L^{1 / 2}=R_{j}^{L} L^{1 / 2}$ and $\bar{Z}_{j}=\bar{Z}_{j} L^{-1 / 2} L^{1 / 2}=$ $\bar{R}_{j}^{L} L^{1 / 2}$, by Proposition 2,

$$
\begin{aligned}
\|f\|_{\mathscr{H}_{L}^{1,1}} & =\left\|L^{1 / 2} f\right\|_{H_{L}^{1}}+\|f\|_{1} \\
& \leq \sum_{j=1}^{n}\left(\left\|R_{j}^{L} L^{1 / 2} f\right\|_{1}+\left\|\bar{R}_{j}^{L} L^{1 / 2} f\right\|_{1}\right)+\|f\|_{1} \\
& =\sum_{j=1}^{n}\left(\left\|Z_{j} f\right\|_{1}+\left\|\bar{Z}_{j} f\right\|_{1}\right)+\|f\|_{1} \\
& \leq \sum_{j=1}^{n}\left(\left\|Z_{j} f\right\|_{H_{L}^{1}}+\left\|\bar{Z}_{j} f\right\|_{H_{L}^{1}}\right)+\|f\|_{1} \\
& \leq C\|f\|_{H_{L}^{1,1}}
\end{aligned}
$$

that is, $f \in \mathscr{H}_{L}^{1,1}\left(\mathbb{C}^{n}\right)$.

If $f \in \mathscr{H}_{L}^{1,1}\left(\mathbb{C}^{n}\right)$, then, by Proposition 3,

$$
\begin{aligned}
\|f\|_{H_{L}^{1,1}} & =\sum_{j=1}^{n}\left(\left\|Z_{j} f\right\|_{H_{L}^{1}}+\left\|\bar{Z}_{j} f\right\|_{H_{L}^{1}}\right)+\|f\|_{1} \\
& =\sum_{j=1}^{n}\left(\left\|R_{j}^{L} L^{1 / 2} f\right\|_{H_{L}^{1}}+\left\|\bar{R}_{j}^{L} L^{1 / 2} f\right\|_{H_{L}^{1}}\right)+\|f\|_{1} \\
& \leq C\left\|L^{1 / 2} f\right\|_{H_{L}^{1}}+\|f\|_{1} \leq C\|f\|_{\mathscr{P}_{L}^{1,1}} .
\end{aligned}
$$

This gives the proof of Theorem 10 .

By Proposition 3 and Theorem 10, we can get the following endpoint case of square root problem for $L$ (for the elliptic second-order divergence operator see Theorem 40 in [10]).

Corollary 11. There exists $C>0$ such that, for all $f \in$ $H_{L}^{1,1}\left(\mathbb{C}^{n}\right)$,

$$
\begin{aligned}
C^{-1}\|f\|_{H_{L}^{1,1}} & \leq\left\|\nabla_{L} f\right\|_{H_{L}^{1}} \leq C\left\|L^{1 / 2} f\right\|_{H_{L}^{1}} \leq C\|f\|_{\mathscr{H}_{L}^{1,1}} \\
& \leq C\|f\|_{H_{L}^{1,1}}
\end{aligned}
$$

In the following, we consider the atomic decomposition of $H_{L}^{1,1}\left(\mathbb{C}^{n}\right)$.
Definition 12. We say a function $b(x)$ is an $(1, q)$-atom associates with a ball $B\left(z_{0}, r\right)$ for the space $H_{\mathrm{L}}^{1,1}\left(\mathbb{C}^{n}\right)$, if

(1) $\operatorname{supp} b \subset B\left(z_{0}, r\right)$,

(2) $\left\|L^{1 / 2} b\right\|_{q} \leq\left\|B\left(z_{0}, r\right)\right\|^{1 / q-1}$,

where $1<q \leq \infty$.

The atomic quasi-norm in $H_{L}^{1,1}\left(\mathbb{C}^{n}\right)$ is defined by

$$
\|f\|_{L \text {-atom }}=\inf \left\{\sum\left|c_{j}\right|\right\},
$$

where the infimum is taken over all decompositions $f=$ $\sum c_{j} b_{j}$, where $b_{j}$ are $(1, q)$-atoms.

In order to prove the atomic decomposition of $H_{L}^{1,1}\left(\mathbb{C}^{n}\right)$, we need the following lemma.

Lemma 13. Let $a(z)$ be a $(1, q)$-atom associated with ball $B\left(z_{0}, r\right)$ of $H_{L}^{1}\left(\mathbb{C}^{n}\right)$ and $b(z)=L^{-1 / 2} a(z)$. Then

$$
\begin{aligned}
& \left|Z_{j} b(z)\right| \leq C \frac{r}{\left|z-z_{0}\right|^{2 n+1}}, \\
& \left|\bar{Z}_{j} b(z)\right| \leq C \frac{r}{\left|z-z_{0}\right|^{2 n+1}}
\end{aligned}
$$

for $\left|z-z_{0}\right| \geq 2 r$ and $j=1,2, \ldots, n$.

Proof. For $j=1,2, \ldots, n$, since $a(z)$ is a $(1, q)$-atom, we have

$$
\begin{gathered}
\left|Z_{j} b(z)\right|=\left|Z_{j} L^{-1 / 2} a(z)\right|=\left|R_{j}^{L} a(z)\right|=\mid \int_{\mathbb{C}^{n}} a(u) \\
\cdot \frac{z_{j}-u_{i}}{|z-u|^{2 n+1}} \psi(z-u) \bar{\omega}(z, u) d u|=| \int_{\mathbb{C}^{n}} a(u) \\
\cdot \bar{\omega}\left(z_{0}, u\right)\left(\frac{z_{j}-u_{i}}{|z-u|^{2 n+1}} \psi(z-u) \omega\left(z_{0}-z, u\right)\right. \\
\left.-\frac{z_{j}-z_{0, j}}{\left|z-z_{0}\right|^{2 n+1}} \psi\left(z-z_{0}\right) \omega\left(z_{0}-z, z_{0}\right)\right) d u \mid,
\end{gathered}
$$

and in the last equality, we use the fact $\bar{\omega}(z, u)=$ $\bar{\omega}\left(z_{0}, u\right) \omega\left(z_{0}-z, u\right)$, the definition of Riesz transform and atom.

when $u \in B\left(z_{0}, r\right)$ and $\left|z-z_{0}\right|>2 r$, we have $|z-u| \sim\left|z-z_{0}\right|$, so

$$
\begin{aligned}
& \left|Z_{j} b(z)\right|=\mid \int_{\mathbb{C}^{n}} a(u) \bar{\omega}\left(z_{0}, u\right) \\
& \cdot\left(\frac{z_{j}-u_{i}}{\left|z-z_{0}\right|^{2 n+1}} \psi(z-u) \omega\left(z_{0}-z, u\right)\right. \\
& \left.\quad-\frac{z_{j}-z_{0, j}}{\left|z-z_{0}\right|^{2 n+1}} \psi\left(z-z_{0}\right) \omega\left(z_{0}-z, z_{0}\right)\right) d u \mid .
\end{aligned}
$$


Let $g(u)=\left(z_{j}-u_{j}\right) \psi(z-u) \omega\left(z_{0}-z, u\right)$. Then there exists a constant $C>0$ such that $\left|g^{\prime}(u)\right| \leq C$. Therefore,

$$
\begin{aligned}
\left|g(u)-g\left(z_{0}\right)\right| & =\left|g^{\prime}(\xi)\right|\left|u-z_{0}\right| \leq C\left|u-z_{0}\right|, \\
\left|Z_{j} b(z)\right| & \leq C \int_{\mathbb{C}^{n}} \frac{\left|u-z_{0}\right|}{\left|z-z_{0}\right|^{2 n+1}}|a(u)| d u \\
& \leq C \frac{r}{\left|z-z_{0}\right|^{2 n+1}} .
\end{aligned}
$$

The proof of $\left|\bar{Z}_{j} b(z)\right|$ is similar to the proof of $\left|Z_{j} b(z)\right|$.

Now we can prove the following result.

Theorem 14. The norms $\|f\|_{H_{L}^{1,1}}$ and $\|f\|_{L \text {-atom }}$ are equivalent; that is, there exists a constant $C>0$ such that

$$
C^{-1}\|f\|_{H_{L}^{1,1}} \leq\|f\|_{L \text {-atom }} \leq C\|f\|_{H_{L}^{1,1}} .
$$

Proof. To show $f=\sum \lambda_{i} b_{i} \in H_{L}^{1,1}\left(\mathbb{C}^{n}\right)$, it suffices to prove that for any $(1, q)$-atom $b$, with $\|b\|_{H_{L}^{1,1}} \leq C$ independent of $b$. By Theorem 10 and Proposition 3,

$$
\begin{aligned}
& \|b\|_{H_{L}^{1,1}}=\left\|L^{1 / 2} b\right\|_{H_{L}^{1}}=\sum_{j=1}^{n}\left(\left\|R_{j} L^{1 / 2} b\right\|_{1}+\left\|\bar{R}_{j} L^{1 / 2} b\right\|_{1}\right) \\
& +\left\|L^{1 / 2} b\right\|_{1}=\sum_{j=1}^{n}\left(\left\|Z_{j} b\right\|_{1}+\left\|\bar{Z}_{j} b\right\|_{1}\right)+\left\|L^{1 / 2} b\right\|_{1} \\
& =\sum_{j=1}^{n}\left(\int_{B\left(z_{0}, r\right)}\left|R_{j} L^{1 / 2} b(z)\right| d z\right. \\
& \left.+\int_{B\left(z_{0}, r\right)}\left|\bar{R}_{j} L^{1 / 2} b(z)\right| d z\right)+\int_{B\left(z_{0}, r\right)}\left|L^{1 / 2} b(z)\right| d z \\
& \leq|B|^{1 / q^{\prime}} \sum_{j=1}^{n}\left(\left\|R_{j} L^{1 / 2} b(z)\right\|_{q}+\left\|\bar{R}_{j} L^{1 / 2} b(z)\right\|_{q}\right) \\
& \cdot|B|^{1 / q^{\prime}}\left\|L^{1 / 2} b(z)\right\|_{q} \leq C|B|^{1 / q^{\prime}}|B|^{1 / q-1} \leq C .
\end{aligned}
$$

For the reverse, if $f \in H_{L}^{1,1}\left(\mathbb{C}^{n}\right)$, there exists $g \in H_{L}^{1}\left(\mathbb{C}^{n}\right)$ such that $f=L^{-1 / 2} g$. Since $g=\sum \lambda_{i} a_{i}$, where $a_{i}$ are $(1, q)$-atoms in $H_{L}^{1}$, we get $f=\sum \lambda_{i} L^{-1 / 2} a_{i}$ with $\sum\left|\lambda_{j}\right|<\infty$. Since $L^{-1 / 2} a_{i}$ does not have compact support, it is not an atom for $H_{L}^{1,1}\left(\mathbb{C}^{n}\right)$.

Let $a$ be a $(1, q)$-atom of $H_{L}^{1}$ such that supp $a \subset B\left(z_{0}, r\right)$ and $b(z)=L^{-1 / 2} a$. We choose a smooth partition of unity $1=\phi_{0}+\sum_{m=1}^{\infty} \phi_{m}$, where $\phi_{0} \equiv 1$ and $\phi_{1} \equiv 0$ on $\left|z-z_{0}\right|<2 r$.

$$
\begin{aligned}
& \operatorname{supp} \phi_{0} \subset\left\{z|| z-z_{0} \mid \leq 4 r\right\}, \\
& \operatorname{supp} \phi_{1} \subset\left\{z|2 r \leq| z-z_{0} \mid \leq 8 r\right\}
\end{aligned}
$$

and $\phi_{m}(z)=\phi_{1}\left(2^{1-m} z\right)$ for $m \geq 2$. Then $b(z)=\phi_{0} b+$ $\sum_{m=1}^{\infty} \phi_{m} b$. We will show $\phi_{m} b=\lambda_{m} b_{m}$ for appropriate scalars $\lambda_{m}$, where $b_{m}$ are $(1, q)$-atoms in $H_{L}^{1,1}\left(\mathbb{C}^{n}\right)$ and $\sum\left|\lambda_{m}\right|<C$.
It is obvious that $\operatorname{supp} b_{m} \subset B\left(z_{0}, 2^{4+m} r\right)$. Let

$$
\lambda_{m}=\left[2^{(4+m)} r\right]^{2 n(1-1 / q)}\left\|L^{1 / 2} \phi_{m} b\right\|_{q} .
$$

For $m=0$, since $\left\|L^{1 / 2} b\right\|_{q}=1$, we get $\left\|L^{1 / 2} \phi_{0} b\right\|_{q} \leq C$. For $m \geq 1$, by Lemma 13 , we have

$$
\begin{aligned}
\left\|Z_{j} \phi_{m} b\right\|_{q} & \leq C\left(2^{m} r\right)^{2 n / q}\left\|Z_{j} \phi_{m} b\right\|_{\infty} \\
& \leq C r\left(2^{m} r\right)^{2 n / q}\left(2^{m} r\right)^{-2 n-1} \\
& =C 2^{-m}\left(2^{m} r\right)^{-2 n / q^{\prime}}, \\
\left\|\bar{Z}_{j} \phi_{m} b\right\|_{q} & \leq C 2^{-m}\left(2^{m} r\right)^{-2 n / q^{\prime}} .
\end{aligned}
$$

So $\lambda_{m} \leq C 2^{-m}$, which gives $\sum\left|\lambda_{m}\right| \leq C$.

In the following, we consider the dual spaces of $H_{L}^{1,1}\left(\mathbb{C}^{n}\right)$. Our proof is motivated by [10].

Definition 15. We say a distribution $T \in \mathscr{D}^{\prime}\left(\mathbb{C}^{n}\right)$ belongs to the BMO-Sobolev spaces $\mathrm{BMO}_{L}^{-1}\left(\mathbb{C}^{n}\right)$ if there exist $\phi_{0} \in$ $L^{\infty}\left(\mathbb{C}^{n}\right)$ and $\Phi \in \mathrm{BMO}_{L}\left(\mathbb{C}^{n}, \mathbb{C}^{n}\right)$ such that $T=\phi_{0}-\operatorname{div} \Phi$. Define

$$
\|T\|_{\mathrm{BMO}_{L}^{-1}\left(\mathbb{C}^{n}\right)}=\inf \left(\left\|\phi_{0}\right\|_{\infty}+\|\Phi\|_{\mathrm{BMO}_{L}\left(\mathbb{C}^{n}\right)}\right),
$$

where the infimum is taken over all functions $\phi_{0} \in L^{\infty}\left(\mathbb{C}^{n}\right)$ and $\Phi \in \mathrm{BMO}_{L}\left(\mathbb{C}^{n}, \mathbb{C}^{n}\right)$ such that $T=\phi_{0}-\operatorname{div} \Phi, \operatorname{div} \Phi=$ $\left(Z_{1} \Phi, \ldots, Z_{n} \Phi, \bar{Z}_{1} \Phi, \ldots, \bar{Z}_{n} \Phi\right)$.

Theorem 16. The dual space of $H_{L}^{1,1}\left(\mathbb{C}^{n}\right)$ is isomorphic to $\mathrm{BMO}_{L}^{-1}\left(\mathbb{C}^{n}\right)$. Moreover, given $T=\phi_{0}-\operatorname{div} \Phi \in \mathrm{BMO}_{L}^{-1}\left(\mathbb{C}^{n}\right)$, the linear functional

$$
\begin{aligned}
& \mathscr{D}\left(\mathbb{C}^{n}\right) \ni f \\
& \longmapsto \int_{\mathbb{C}^{n}} f(z) \phi_{0}(z) d z \\
& \quad+\sum_{j=1}^{n} \int_{\mathbb{C}^{n}}\left(Z_{j} f(z) \Phi_{j}(z)+\bar{Z}_{j} f(z) \Phi_{j}(z)\right) d z \\
& =\langle T, f\rangle
\end{aligned}
$$

extends to a bounded linear functional $L_{T}$ on $H_{L}^{1,1}\left(\mathbb{C}^{n}\right)$. Conversely, for any $L \in\left(H_{L}^{1,1}\left(\mathbb{C}^{n}\right)\right)^{\prime}$, there exists a unique $T \in$ $\mathrm{BMO}_{L}^{-1}\left(\mathbb{C}^{n}\right)$ such that, for all $f \in \mathscr{D}\left(\mathbb{C}^{n}\right), L(f)=\langle T, f\rangle$, one has $\left\|L_{T}\right\| \sim\|T\|_{\mathrm{BMO}_{L}^{-1}\left(\mathbb{C}^{n}\right)}$.

Proof. Let $T \in \mathrm{BMO}_{L}^{-1}\left(\mathbb{C}^{n}\right), \epsilon>0$ and $\phi_{0} \in L^{\infty}\left(\mathbb{C}^{n}\right)$ and $\Phi \in \mathrm{BMO}_{L}\left(\mathbb{C}^{n}, \mathbb{C}^{n}\right)$ such that $T=\phi_{0}-\operatorname{div} \Phi$ and

$$
\left\|\phi_{0}\right\|_{\infty}+\|\Phi\|_{\mathrm{BMO}_{L}\left(\mathbb{C}^{n}\right)} \leq(1+\epsilon)\|T\|_{\mathrm{BMO}_{L}^{-1}\left(\mathbb{C}^{n}\right)} .
$$


Then, for all $f \in \mathscr{D}\left(\mathbb{C}^{n}\right)$,

$$
\begin{aligned}
& |\langle T, f\rangle| \\
& \leq\left|\int_{\mathbb{C}^{n}} f(z) \phi_{0}(z) d z\right| \\
& \quad+\sum_{j=1}^{n}\left|\int_{\mathbb{C}^{n}}\left(Z_{j} f(z) \Phi_{j}(z)+\bar{Z}_{j} f(z) \Phi_{j}(z)\right) d z\right| \\
& \leq\left(\inf \left(\left\|\phi_{0}\right\|_{\infty}+\|\Phi\|_{\mathrm{BMO}_{L}\left(\mathbb{C}^{n}\right)}\right)\right)\|f\|_{H_{L}^{1,1}} .
\end{aligned}
$$

Since $\mathscr{D}\left(\mathbb{C}^{n}\right)$ is dense in $H_{L}^{1,1}\left(\mathbb{C}^{n}\right)$, this means that $f \mapsto\langle T, f\rangle$ extends to a bounded linear form $L_{T}$ on $H_{L}^{1,1}\left(\mathbb{C}^{n}\right)$, with

$$
\left\|L_{T}\right\| \leq(1+\epsilon)\|T\|_{\mathrm{BMO}_{L}^{-1}\left(\mathbb{C}^{n}\right)}
$$

Since this is true for all $\epsilon>0$, one obtains $\left\|L_{T}\right\| \leq$ $\|T\|_{\mathrm{BMO}_{L}^{-1}\left(\mathbb{C}^{n}\right)}$.

Conversely, let $L$ be a bounded linear form on $H_{L}^{1,1}\left(\mathbb{C}^{n}\right)$. Since $H_{L}^{1,1}\left(\mathbb{C}^{n}\right)$ is isometrically isomorphic to a subspace of $L^{1}\left(\mathbb{C}^{n}\right) \oplus H_{L}^{1}\left(\mathbb{C}^{n}\right) \oplus \cdots \oplus H_{L}^{1}\left(\mathbb{C}^{n}\right)$, there exist $\phi_{0} \in L^{\infty}\left(\mathbb{C}^{n}\right)$ and $\phi_{1}, \ldots, \phi_{d} \in \mathrm{BMO}_{L}\left(\mathbb{C}^{n}\right)$ such that, for all $f \in \mathscr{D}\left(\mathbb{C}^{n}\right)$,

$$
\begin{aligned}
& L(f) \\
& \quad=\int_{\mathbb{C}^{n}} f(z) \phi_{0}(z) d z \\
& \quad+\sum_{j=1}^{n} \int_{\mathbb{C}^{n}}\left(Z_{j} f(z) \Phi_{j}(z)+\bar{Z}_{j} f(z) \Phi_{j}(z)\right) d z, \\
& \sup _{1 \leq j \leq n}\left(\left\|\phi_{0}\right\|_{\infty},\left\|\Phi_{j}\right\|_{\mathrm{BMO}_{L}}\right) \leq\|L\| .
\end{aligned}
$$

Set $\Phi=\left(\phi_{1}, \ldots, \phi_{n}\right)$ and $T=\phi_{0}-\operatorname{div} \Phi \in \mathscr{D}^{\prime}\left(\mathbb{C}^{n}\right)$. Then $T \in \mathrm{BMO}_{L}^{-1}\left(\mathbb{C}^{n}\right), L=L_{T}$ and $\|T\| \leq(2 n+1)\|L\|$. This proves Theorem 16.

\section{An Application: Div-Curl Lemma}

In [13], the authors proved the following well-known divcurl Lemma: Let $1<p, q<\infty$ and $1 / p+1 / q=1$. If $f \in L^{p}\left(\mathbb{R}^{d}, \mathbb{R}^{d}\right)$ with curlf $=0$ and $e \in L^{q}\left(\mathbb{R}^{d}, \mathbb{R}^{d}\right)$ with $\operatorname{div} e=0$ on $\mathbb{R}^{d}$, then $e \cdot f \in H^{1}\left(\mathbb{R}^{d}\right)$. Now, we consider the case of $p=1$; as an application of Theorem 14, we give the endpoint version of the div-curl lemma.

Theorem 17. Let $f \in H_{L}^{1,1}\left(\mathbb{C}^{n}\right)$ and $e \in L^{\infty}\left(\mathbb{C}^{n}, \mathbb{C}^{n}\right)$ with $\operatorname{div} e=0$ on $\mathbb{C}^{n}$. Then $e \cdot \nabla_{L} f \in H_{L}^{1}\left(\mathbb{C}^{n}\right)$, where $\nabla_{L}=$ $\left(Z_{1}, \ldots, Z_{n}, \bar{Z}_{1}, \ldots, \bar{Z}_{n}\right)$.

Proof. If $f \in H_{L}^{1,1}\left(\mathbb{C}^{n}\right)$, by Theorem 14, $f$ has the decomposition

$$
f=\sum_{i=0}^{\infty} \lambda_{i} a_{i}
$$

where the $a_{k}$ are $H_{L}^{1,1}$-atoms and $\sum_{k=0}^{\infty}\left|\lambda_{i}\right|<\infty$. Therefore, for $e \in L^{\infty}\left(\mathbb{C}^{n}, \mathbb{C}^{n}\right)$,

$$
e \cdot \nabla_{L} f=\sum_{i=0}^{\infty} \lambda_{i} e \cdot \nabla_{A} a_{i} .
$$

To prove $e \cdot \nabla_{L} f \in H_{L}^{1}\left(\mathbb{C}^{n}\right)$, we need only to prove $e$. $\nabla_{L} a_{i}$ are $H_{L}^{1}$-atoms by the atomic decomposition of $H_{L}^{1}\left(\mathbb{C}^{n}\right)$. Since $a_{i}$ is an $H_{L}^{1,1}$-atom, there exists a ball $B_{i}\left(z_{0}, r\right)$ such that suppe $\cdot \nabla_{L} a_{i} \subset B_{i}$ and $\left\|\nabla_{L} a_{i}\right\|_{2} \leq\left|B_{i}\right|^{-1 / 2}$. Following from $e \in L^{\infty}\left(\mathbb{C}^{n}, \mathbb{C}^{n}\right)$,

$$
\left\|e \cdot \nabla_{L} a_{i}\right\|_{2} \leq C\left|B_{i}\right|^{-1 / 2} .
$$

Since $a_{i}$ satisfies the moment condition and $\operatorname{div} e=0$, we get $e \cdot \nabla_{L} a_{i}=\operatorname{div}\left(a_{i} e\right)$, and this yields the moment condition

$$
\int_{\mathbb{C}^{n}} e \cdot \nabla_{L} a_{i} \bar{\omega}\left(z, z_{0}\right) d z=0 .
$$

We complete the proof of Theorem 17.

\section{Conflicts of Interest}

The authors declare that there are no conflicts of interest regarding the publication of this article.

\section{Acknowledgments}

This work was supported by the National Natural Science Foundation of China (Grant no. 11471018).

\section{References}

[1] D. Goldberg, "A local version of real Hardy spaces," Duke Mathematical Journal, vol. 46, no. 1, pp. 27-42, 1979.

[2] C. Fefferman and E. M. Stein, "Hp spaces of several variables," Acta Mathematica, vol. 129, no. 3-4, pp. 137-193, 1972.

[3] J. Peetre, “The Weyl transform and Laguerre polynomials," Le Matematiche, vol. 27, pp. 301-323 (1973), 1972.

[4] H. Triebel, Theory of Function Spaces, vol. 78 of Monographs in Mathematics, Birkhäuser, Boston, Mass, USA, 1983.

[5] R. S. Strichartz, " $H^{p}$ Sobolev spaces," Colloquium Mathematicum, vol. 60-61, no. 1, pp. 129-139, 1990.

[6] A. Torchinsky, "Restrictions and extensions of potentials of $H^{p}$ distributions," Journal of Functional Analysis, vol. 31, no. 1, pp. 24-41, 1979.

[7] A. Miyachi, "Hardy-Sobolev spaces and maximal functions," Journal of the Mathematical Society of Japan, vol. 42, no. 1, pp. 73-90, 1990.

[8] R. A. DeVore and R. C. Sharpley, "Maximal functions measuring smoothness," Memoirs of the American Mathematical Society, vol. 47, no. 293, 1984.

[9] D.-C. Chang, G. Dafni, and E. M. Stein, "Hardy spaces, BMO, and boundary value problems for the Laplacian on a smooth domain in $R^{n}$," Transactions of the American Mathematical Society, vol. 351, no. 4, pp. 1605-1661, 1999.

[10] P. Auscher, E. Russ, and P. Tchamitchian, "Hardy Sobolev spaces on strongly Lipschitz domains of $R^{n}$," Journal of Functional Analysis, vol. 218, no. 1, pp. 54-109, 2005. 
[11] P. Koskela and E. Saksman, "Pointwise characterizations of Hardy-Sobolev functions," Mathematical Research Letters, vol. 15, no. 4, pp. 727-744, 2008.

[12] Z. Lou and S. Yang, "An atomic decomposition for the HardySobolev space," Taiwanese Journal of Mathematics, vol. 11, no. 4, pp. 1167-1176, 2007.

[13] R. Coifman, P.-L. Lions, Y. Meyer, and S. Semmes, "Compensated compactness and Hardy spaces," Journal de Mathématiques Pures et Appliquées, vol. 72, no. 3, pp. 247-286, 1993.

[14] Y.-K. Cho and J. Kim, "Atomic decomposition on HardySobolev spaces," Studia Mathematica, vol. 177, no. 1, pp. 25-42, 2006.

[15] S. Janson, "On functions with derivatives in $H^{1}$," in Harmonic analysis and partial differential equations (El ESCorial, 1987), vol. 1384 of Lecture Notes in Math., pp. 193-201, Springer, Berlin, 1989.

[16] J. Orobitg, "Spectral synthesis in spaces of functions with derivatives in $H^{1}$," in Harmonic analysis and partial differential equations (El ESCorial, 1987), vol. 1384 of Lecture Notes in Math., pp. 202-206, Springer, Berlin, 1989.

[17] P. K. Ratnakumar and V. K. Sohani, "Nonlinear Schrödinger equation and the twisted Laplacian-global well posedness," Mathematische Zeitschrift, vol. 280, no. 1-2, pp. 583-605, 2015.

[18] G. Mauceri, M. A. Picardello, and F. Ricci, "A Hardy space associated with twisted convolution," Advances in Mathematics, vol. 39, no. 3, pp. 270-288, 1981.

[19] R. F. V. Anderson, "The multiplicative Weyl functional calculus," Journal of Functional Analysis, vol. 9, no. 4, pp. 423-440, 1972.

[20] A. Grossmann, G. Loupias, and E. M. Stein, "An algebra of pseudodifferential operators and quantum mechanics in phase space," Université de Grenoble. Annales de l'Institut Fourier, vol. 18, pp. 343-368, viii (1969), 1968.

[21] J. Huang and Y. Liu, "Molecular characterization of Hardy spaces associated with twisted convolution," Journal of Function Spaces, Article ID 326940, Art. ID 326940, 6 pages, 2014.

[22] E. M. Stein, Singular Integrals and Differentiability Properties of Functions, Princeton University Press, Princeton, NJ, USA, 1970. 


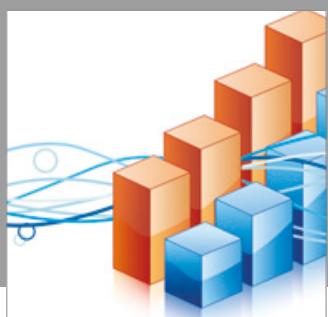

Advances in

Operations Research

vatersals

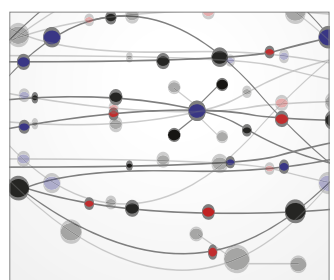

\section{The Scientific} World Journal
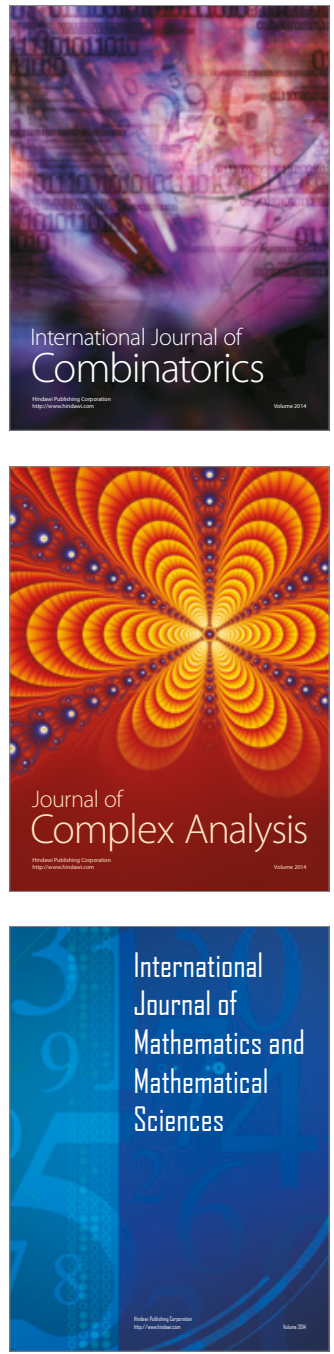
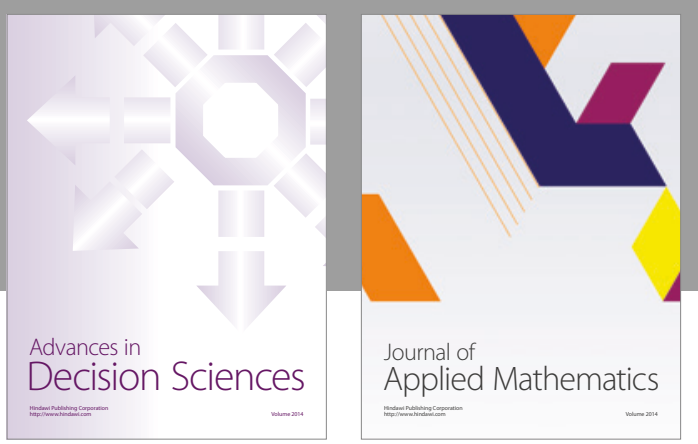

Algebra

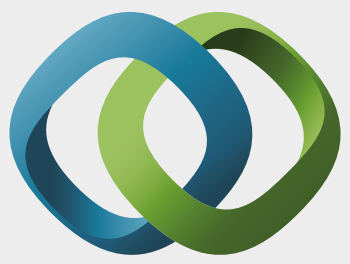

\section{Hindawi}

Submit your manuscripts at

https://www.hindawi.com
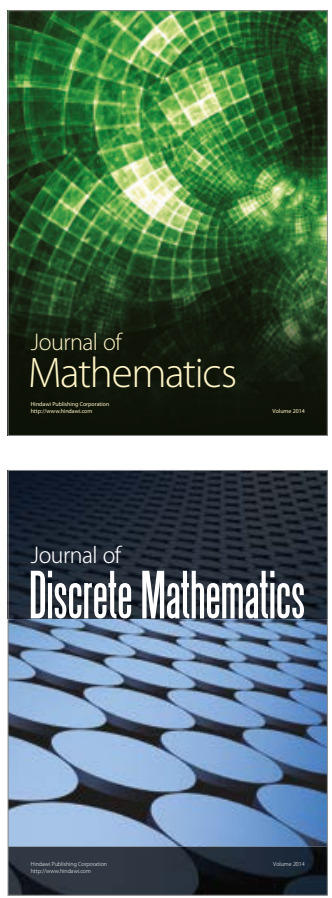

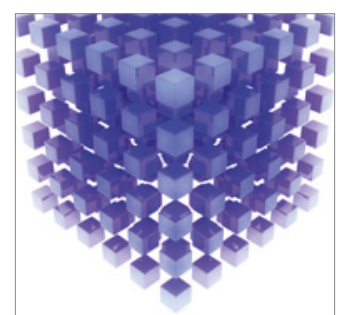

Mathematical Problems in Engineering
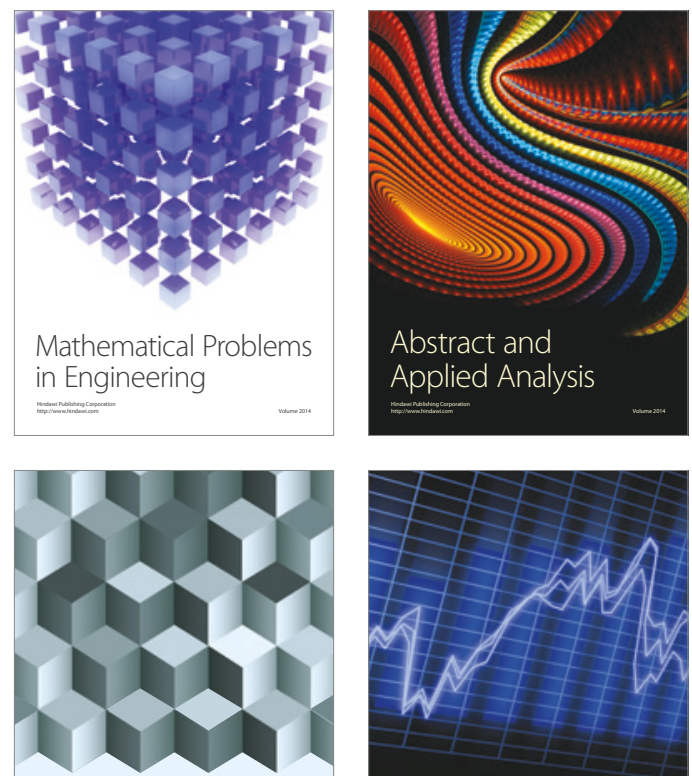

Journal of

Function Spaces

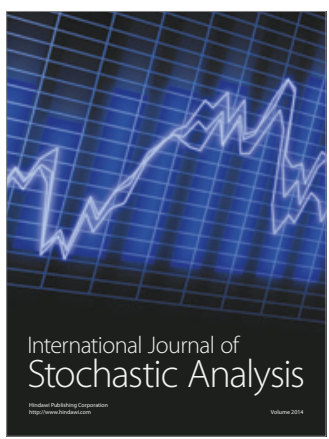

Probability and Statistics
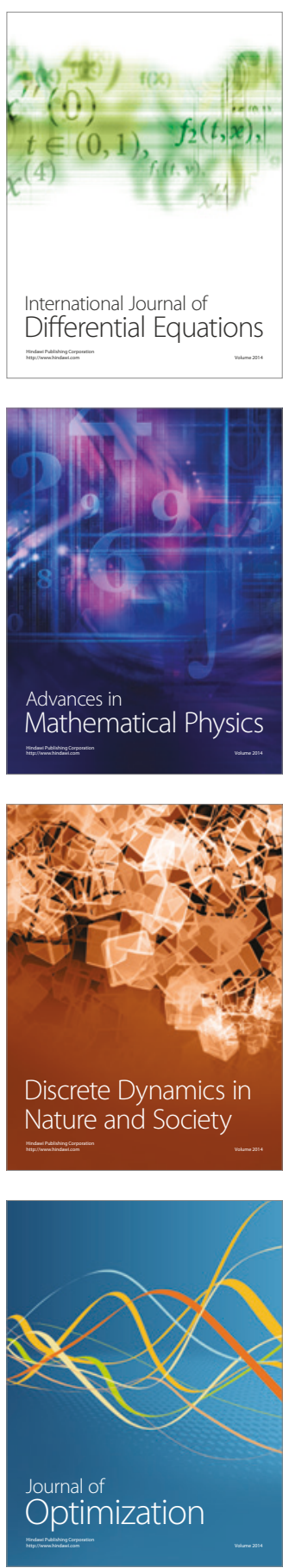